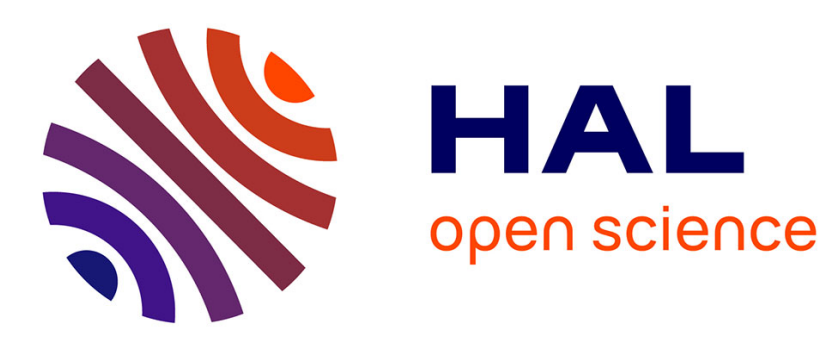

\title{
Epileptic seizures identification with autoregressive model and firefly optimization based classification
}

\author{
Abdelouahab Attia, Abdelouahab Moussaoui, Youssef Chahir
}

\section{To cite this version:}

Abdelouahab Attia, Abdelouahab Moussaoui, Youssef Chahir. Epileptic seizures identification with autoregressive model and firefly optimization based classification. Evolving Systems, 2021, 12 (3), pp.827-836. 10.1007/s12530-019-09319-z . hal-02456493

HAL Id: hal-02456493

https://hal-normandie-univ.archives-ouvertes.fr/hal-02456493

Submitted on 27 Jan 2020

HAL is a multi-disciplinary open access archive for the deposit and dissemination of scientific research documents, whether they are published or not. The documents may come from teaching and research institutions in France or abroad, or from public or private research centers.
L'archive ouverte pluridisciplinaire HAL, est destinée au dépôt et à la diffusion de documents scientifiques de niveau recherche, publiés ou non, émanant des établissements d'enseignement et de recherche français ou étrangers, des laboratoires publics ou privés. 


\title{
Epileptic seizures identification with autoregressive model and firefly optimization based classification
}

\author{
${\text { Abdelouahab } \text { Attia }^{1} \text { (D) Abdelouahab Moussaoui }}^{2} \cdot$ Youssef Chahir $^{3}$
}

Received: 17 February 2019 / Accepted: 7 December 2019

\begin{abstract}
Identifying epilepsy cases and epileptic seizures from electroencephalogram (EEG) signals is a challenging issue, which usually needs high level of skilled neurophysiologists. Numerous works have attempted to develop tools that can provide an assistant to neurophysiologist in analyzing the EEG for epileptic seizures detection. This paper proposes a new automatic framework to identify and classify the epileptic seizure from EEG using a machine learning method. In particular, the feature extraction process of the proposed scheme utilizes autoregressive model (AR) and firefly optimization (FA) to procure an optimal model order $(\mathrm{P})$. Namely, the main aim of FA is to find the best model order $(\mathrm{P})$ with minimum residual variance using Akaike information criterion (AIC) as an objective function of FA algorithm. A support vector machine (SVM) classifier is employed for the classification of the epileptic seizures signals. The presented scheme is also effective for short segment of EEG signals owing to use of AR model in features extraction stage. Experiments with the publicly available Bonn database that is composed of healthy (nonepileptic), interictal and ictal EEG samples show promising results with high accuracy.
\end{abstract}

Keywords Epileptic seizures classification $\cdot$ AR model $\cdot$ Firefly algorithm $\cdot$ SVM $\cdot$ Akaike information criterion

\section{Introduction}

Epilepsy is a chronic brain disorder marked by sudden recurrent episodes of sensory disturbance, loss of consciousness, or convulsions, associated with abnormal electrical activity in the brain. Diagnosis of epileptic disease using electroencephalogram (EEG) is a challenge task in the field of biomedical science, which is mainly performed by a very skilled neurophysiologist (Chiarelli et al. 2017; Attia et al. 2017). The EEG signals are the electrical activity recorded from the

Abdelouahab Attia

attia.abdelouahab@gmail.com

Abdelouahab Moussaoui

moussaoui.abdel@gmail.com

Youssef Chahir

youssef.chahir@unicaen.fr

1 Computer Science Department, Faculty of Mathematics and Informatics, Mohamed El Bachir El Ibrahimi University of Bordj Bou Arreridj, 34000 Bordj Bou Arreridj, Algeria

2 Computer Science Department, Faculty of Sciences, Ferhat Abbas University Setif I, Setif, Algeria

3 Image Team GREYC, CNRS, UMR 6072, University of Caen, Caen, France scalp of the human brain to be analyzed by neurophysiologists for predicting and identifying epileptic diseases; especially detecting and classifying epileptic seizures. The EEG analysis for epileptic seizures has received considerable attention in the biomedical research field in the past decade (Belhadj et al. 2016a; Übeyli 2008a; Amorim et al. 2017). In addition, diagnosis of epileptic based on automated and intelligent systems detection have become vital of brain analysis, which involves high classification schemes (Angelov and Kasabov 2006; Angelov and Zhou 2008; Belhadj et al. 2016b).

In recent years, several machine learning-based methods have been proposed in the literature to classify EEG signals for identification of epileptic seizures. For instance, Tezel et al. (Tezel et al. 2009) introduced a framework using artificial neural network (ANN) and relative wavelet energy. Swami et al. (2016) used hand-crafted features including Shannon entropy, standard deviation, and energy, which are considered a result of extraction. The general regression neural network (GRNN) classifier has been employed in classification step. Guo et al. (2010) have proposed a framework based on line length feature and ANN method in order to identifying automatic epileptic seizure. Wang et al. (2011) proposed a scheme based on wavelet packet and K-Nearest Neighbor (KNN) as well as support vector machine (SVM) classifiers for epileptic 
seizures classification. Chandaka et al. (2009) have been used Cross-correlation with SVM classifier in classification of EEG signals. The Authors in Übeyli (2008b) presented a method that employed the multiclass support vector machine (SVM) for EEG signal classification. Few works have also exploited dimensionality reduction techniques such as Linear discriminant analysis (LDA), Principle Component Analysis (PCA), Singular value decomposition (SVD), and Independent Component Analysis (ICA) before classification of EEG signals (Subasi and Gursoy 2010). Nicolaou and Georgiou (2012) have been proposed a scheme based on the permutation entropy and SVM used to detect epileptic seizures.

Nowadays, Autoregressive model (AR) alongside machine learning methods have been used in analyzing times series (Padmavathi and Ramakrishna 2015a; Ansari et al. 2018; Ordóñez et al. 2019). More recently, AR model has been used to generate features for the EEG signals (Shiman et al. 2012; Zhang et al. 2017). The AR model provided parameters that are directly useable by classifier algorithms. The AR is used owing to its ability to effectively process the high frequency resolution and the smoother spectra when the signals are divided into set of segments; especially the EEG signal that are considered as a no stationary signal (Sharma and Pachori 2015). However, to extract significant features of the signals using AR model, two types of feature extraction methods have been practiced: parametric method and non-parametric method (Fabri et al. 2011). Also, Evolutionary based approaches have been utilized in the classification of epileptic seizure (Atyabi et al. 2012). Representative examples of studies that have applied the evolutionary methods are Genetic Algorithm (GA) (Erguzel et al. 2015), Ant Colony Optimization (ACO), (Khushaba et al. 2008), Particle Swarm Optimization (PSO) (Yalcin et al. 2015) and firefly optimization (FA).

Despite the latest progress in detecting epileptic seizures, the error rates are not low enough (Padmavathi and Ramakrishna 2015b). Moreover, majority of prior works have fewer efficacies for short EEG episodes (Burg 1968). There is ample room to devise novel framework to classify epileptic seizures using EEG signals. Therefore, in this paper, we propose a scheme to classify epileptic seizure more accurately from EEG signals. In the feature extraction stage, Burg AR model and FA algorithm have been employed to obtain the best model order $\mathrm{P}$ to obtain the best autoregressive coefficients with minimum noise variance. The features are then fed to the SVM method for final classification. It is worth noticing that one of the common problems in AR methods is the choice of an adequate AR model order known as (P model order) (Krusienski et al. 2006). To address this issue, in this work, the AR parameters have been generated using Burg algorithm with evaluation of the residual modelling error with FA method selecting $\mathrm{P}$ model order, such that Akaike Information Criterion (AIC) function (Akaike 1974) has been used as an objective function by FA algorithm. Experimental analysis on publicly available Bonn dataset collected from healthy no-epileptic and epileptic volunteers (interictal/ictal) with eyes' tasks (closed/open) demonstrate the effectiveness of the proposed framework with high accuracy.

The remainder of this paper is organized as follows. Section 2 presents the proposed approach. Experimental protocol, dataset, figures of merit, experimental results are presented in Sect. 3. Finally, conclusions are drawn in Sect. 4.

\section{Proposed methodology}

Figure 1 shows the schematic diagram of the proposed framework for epileptic seizures classification using EEG signals. The framework is composed of following steps: feature extraction using Burg method (AR-B) hybrid technique with firefly method to get the adequate model order $\mathrm{P}$ that is used in selecting the best coefficients provided from AR model, and finally an SVM classification method. Below, we give details of autoregressive modeling for EEG, Burg algorithm, Akaike information criterion, optimization AR model order $\mathrm{P}$ by firefly algorithm (AR-FA) and support vector machines.

\subsection{Autoregressive modelling for EEG}

AR model has become one of the prominent parametric methods that have been applied in many studies to model EEG signals. AR model permits describing the EEG signal as a linear representation. The regression model of the EEG signal has been accomplished by using the following formula (Zhang et al. 2017; Akaike 1974):

$X(n)=-\sum_{k=1}^{p} a_{k} x(n-k)+e(n)$

where $a_{k}$ represents the AR parameters, $P$ stands for the given model order and $e(n)$ denotes the error term independent of previous samples assumed to be white Gaussian noise with zero mean and variance $\sigma^{2}$.

\subsection{Burg algorithm}

The Burg Algorithm has been utilized to estimate the parameters of an AR model because it is different for other

Fig. 1 Scheme of the proposed framework

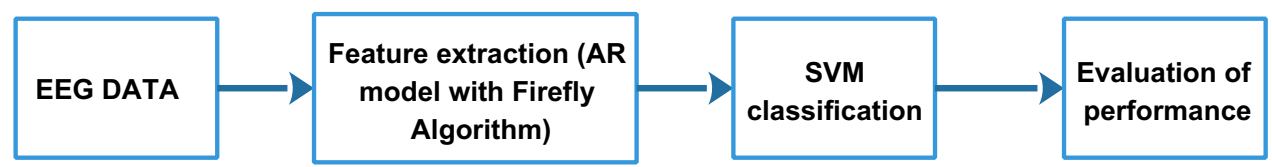


methods that guarantee to generate a stable model. Assume that the input signal EEG $\mathrm{X}(\mathrm{n}) ; n=1,2,3, \ldots ., N$, where $N$ refer to the size of EEG signal. The algorithm is a recursive method based on the lattice filter structure in order to minimize the forward and backward prediction error and it is executed in four steps as follows (Burg 1968):

Step 1. Calculate the initial values of error variance, i.e., computing forward error and backward error by the following equations, respectively:

$\sigma^{2}(0)=\frac{1}{N} \sum_{0}^{N-1}(X(n))^{2}$

$e_{n}(0)=X(n)$

$b_{n-1}(0)=X(n-1)$

Step 2. Calculate reflection coefficient and error variance by the given equations, respectively:

$\pi_{m}=\frac{\sum_{n=m}^{N-1} b_{n-1}(m-1) e_{n}(m-1)}{\sum_{n=m}^{N-1}\left(e_{n}^{2}(m-1)+b_{n-1}^{2}(m-1)\right)}$

$\sigma^{2}(m)=\left(1-\left|\pi_{m}\right|^{2}\right) \sigma^{2}(m-1)$

Step 3. Update Error and AR coefficients:

$\left\{\begin{array}{c}a_{k}(m)=a_{k}(m-1)+\pi_{m} a_{m-k}(m-1) \text { if } m>1 \\ a_{m}(m)=\pi_{1} \text { if } m=1\end{array}\right.$

Forward error:

$e_{n}(m)=e_{n}(m-1)+\pi_{m} b_{n-1}(m-1)$

Backward error:

$b_{n}(m)=b_{n-1}(m-1)+\pi_{m} e_{n}(m-1)$

Step 4. Repeat steps 2 and 3 (with $\mathrm{m}$ incremented by one) until the selected model order $\mathrm{p}$ is reached.

\subsection{The Akaike information criterion}

Recently, The Akaike information criterion (AIC) (Akaike 1974) has been employed in evaluation of the AR model coefficients. However, the AIC method permits selecting an adequate AR model order P (Ouelli et al. 2015). Assuming that the input has Gaussian statistics, the AIC for an AR process is defined by:
$A I C(p)=\ln \left(\sigma^{2}\right)+\frac{2 p}{N}$

where $\sigma^{2}$ is white noise variance. $\mathrm{P}$ is model order and $\mathrm{N}$ represents the length of EEG signal.

\subsection{Optimization AR model order P by firefly algorithm (AR-FA)}

One of the main objectives in this study is identifying an adequate model order $\mathrm{P}$, which is also one of the common problems in AR methods (Krusienski et al. 2006). Hence, the AR Burg algorithm has been used to generate the coefficients and evaluation of the residual modelling error by the (AIC) metric (Akaike 1974). The optimal model order $\mathrm{P}$ that gives the minimum of $\mathrm{AIC}(\mathrm{P})$ has been calculated by (Eq. 10). For that reason, Firefly Optimization method has been employed in order to get the optimal model order P.

In order to generate the AR coefficients $\left(a_{i}\right)$ using EEG signal $\mathrm{X}(\mathrm{n}), n=1,2,3, \ldots ., \mathrm{N}$, we need to fix the model order $\mathrm{P}$, where $\mathrm{P}$ belong in $\{4,5, \ldots, \mathrm{N} / 3$. $\}$. Then, AR method computes the $\left(\mathrm{a}_{\mathrm{i}}\right)$ coefficients with $\sigma^{2}$ white noise variance. However, the major issue is the choice of model order $\mathrm{P}$ that permits achieving an optimal value of AIC (P). For this reason, FA method has been employed with the AIC (P) as an objective function to find the best model order P. The mathematical formulation of the optimization problem that has been focused in this work is given as follows:

$\min _{P \in 4 \ldots N / 3}(\operatorname{AIC}(P))$

where $\mathrm{AIC}(\mathrm{P})$ is a continuous nonlinear objective function.

\subsubsection{FA algorithm}

To construct the mathematical model of the algorithm, the Firefly algorithm is based on three basic rules (Yang 2009):

1. All fireflies are unisex, involving that all fireflies can attract each other regardless of their sex.

2. The attractiveness between fireflies is proportional to their brightness. The firefly with less bright will move towards the brighter one. If no one is brighter than a particular firefly, it moves randomly. Attractiveness is relative to the brightness that reduces with rising distance between fireflies.

3. The brightness known as light intensity of a firefly is relying on the objective function. In application (simulation), the brightness of every firefly is directly relative to the value of the objective function.

The FA algorithm is based on two principals' ideas, the light intensity emitted and the degree of attractiveness. 
The light intensity Ii varies with the distance $r_{i j}$ monotonically and exponentially. That is given as:

$I_{i}=e^{-\gamma r_{i j}}$

where $\gamma$ stand for the light absorption coefficient generally in practice taken as land the distance $r_{i j}$ between two fireflies $\mathrm{i}$ and $\mathrm{j}$ given as:

$r_{i j}=x_{i}-x_{j 2}$

The attractiveness $\beta_{i j}$ of the firefly depends on both the light intensity seen by an adjacent firefly and its distance, that may be represented by:

$\beta_{i j}=\beta_{0} e^{-\gamma r_{i j}^{2}}$

where $\beta_{0}$ is the attractiveness at $r_{i j}=0$.

The amount of movement of firefly $i$ towards to another more attractive firefly $\mathrm{j}$ is given by:

$x_{i}=x_{i}+\beta_{i j}\left(x_{j}-x_{i}\right)+\alpha \varepsilon_{i j}$

where $\varepsilon_{i j}$ is a random parameter generated by a uniform distribution and $\alpha$ is a parameter of scale.
The pseudo code of the firefly algorithm can be summarized as below:

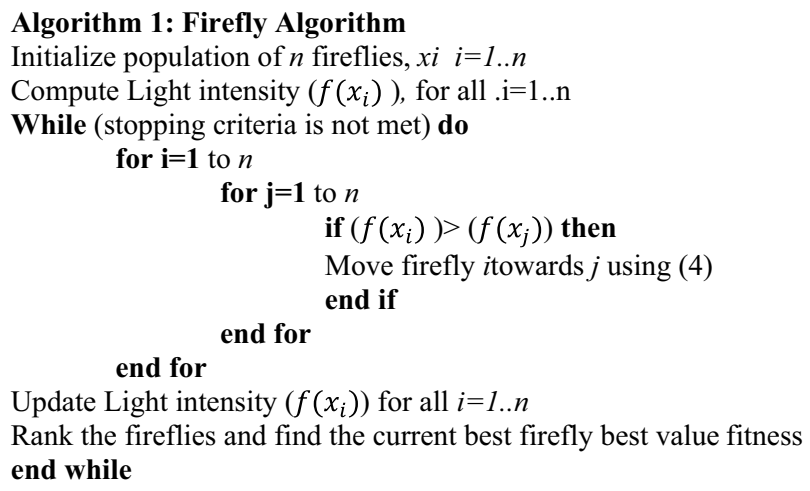

\subsubsection{Model order FA algorithm}

This subsection describes the use of Firefly method in the step of feature extraction. The AR-FA selects an optimal model order that chooses best parameters. The scheme and pseudo code of AR-FO are illustrated in the following::

\section{Algorithm AR-FA}

Input: EEG signal, [minp,maxp] range of order p, np: number of fireflies, it_max: max_iteration

Output: The model order with AIC and minimum noise variance (NSV) is given as:[P, AIC NSV]

begin

for $r=1$ to $n p$

initialize the fireflies with random model order value

end for

while not stop criterion do

$$
\begin{aligned}
& \text { for } i=1 \text { to } n p \text { do } \\
& \text { for } \mathrm{j}=1 \text { to } n p \text { do } \\
& \text { compute }\left(f\left(x_{i}\right), f\left(x_{j}\right)\right) \text { value using AR burg model and AIC functions (provided by Matlab } \\
& \text { known as } \operatorname{arburg}(\mathrm{x}, \mathrm{p}) \mathrm{AIC}(\mathrm{p})) \text { respectively } \\
& \text { if }\left(f\left(x_{i}\right)>f\left(x_{j}\right)\right) \text { then } \\
& \text { Move firefly itowards } j \text { using (4) } \\
& \text { end if } \\
& \text { end for } \\
& \text { end for } \\
& \text { fori }=1: n p \text { do }
\end{aligned}
$$

Update Light intensity $\left(f\left(x_{i}\right)\right)$

end for

Rank the fireflies and update velocity and position of the fireflies and find the current best firefly with $\min (\mathrm{AIC}(\mathrm{P}))$

end while 


\subsection{Support vector machines (SVM)}

Support vector machines is a supervised learning method introduced by Cortes and Vapnik (1995). SVM is a powerful classifier algorithm that has been used in different areas, including speech disorder detection, face recognition, cancer identification, seizure prediction and EEG signal classification (Übeyli 2008a). There are three types of support vector machine techniques (Byun and Lee 2002): (i) Linearly separable, (ii) Linearly Inseparable and (iii) Non-linearly separable.

The basic idea of SVM algorithm is based on kernel functions via solving a quadratic optimization problem in separating data into different groups. As a result, SVM method generates the optimal hyperplane with the largest margin. In other words, SVM projects data into another higherdimensional space. Then, SVM traces the optimal hyperplane in the projection space (Übeyli 2010). For the problem being studied in this paper, single hyperplane is sufficient to split the data into two classes, i.e., (+1) represents the first class and $(-1)$ stands for the second class. Furthermore, the choice of kernel function and parameters of SVM plays an important role in the classification performance. Thus, in this work, radial basis functions (RBF) have been used as kernel functions of SVM classifier that achieved acceptable results. The RBF is expressed by:

$K(x, y)=\exp ^{\left(-\frac{|x-y|^{2}}{2 \sigma^{2}}\right)}$

\section{Experiments}

Here, we provide an experimental evaluation of the proposed epileptic seizures detection framework.

\subsection{Dataset}

The Bonn EEG dataset collected by Andrzejak et al. (2001) was used in this work. It has been widely used in epilepsy study and seizure detection and classification and is available online: (http://epileptologie-bonn.de/cms/upload/workg roup/lehnertz/eegdata.html).

The complete data consists of five sets $(\mathrm{Z}, \mathrm{O}, \mathrm{N}, \mathrm{F}, \mathrm{S})$. Each set has 100 single channel EEG segment of $23.6 \mathrm{~s}$ duration. These collections are composed of three groups. The first one is presented by the two sets $\mathrm{Z}$ and $\mathrm{O}$ recorded from five subjects with healthy volunteers (nonepileptic), whereas the set $\mathrm{Z}$ are recorded with condition eyes open. The set $\mathrm{O}$ recorded with eyes closed by using the standard 10-20 electrode placement scheme. The second one interictal group is given by the sets $\mathrm{N}, \mathrm{F}$ originated from the EEG archive of pre-surgical diagnosis set $\mathrm{N}$ recorded from the hippocampal formation of the opposite hemisphere. The set $\mathrm{F}$ is recorded from epileptogenic zone while the third ictal group is represented by the set $\mathrm{S}$ (seizure activity) (see Fig. 2).

All these EEG signals are digitized at 173.61 samples per second using 12-bit resolution. Band-pass filter settings were $0.53-40 \mathrm{~Hz}(12 \mathrm{~dB} / \mathrm{oct})$. Thus, the length of each recording is $173.61 \times 23.6 \approx 4097$ samples. Figure 3 presents an example for each set.

\subsection{Experimental protocol and figures-of-merit}

This study followed the same protocol used in Übeyli (Übeyli 2008a), Subasi and Gursoy (2010), Nicolaou and Georgiou (2012), Yalcin et al. (2015) such that data sets have been divided into three classes: (i) healthy (nonepileptic)-Z, O; (ii) Interictal-N, F; and (iii) Ictal-S. Two directions of experiments have been applied. The first direction followed below steps: EEG the features extraction using AR model without AR-FA method then SVM classifier. The second direction is as follows: the FA has been employed in the step of features extraction with AR model. The AR-FA has been used to get the best AR coefficient that is followed by SVM classifier.

The performance was evaluated via Accuracy (ACC), sensitivity (SEN) and specificity (SPE). The sensitivity describes the true positive ratio, specificity is referred to as true negative ratio, while the accuracy is the ratio of true positive and negative divided by the total number of cases. In particular, TP is true positive results of a classifier; it refers to the number of EEG signals that have epileptic seizure. TN is true negative results denote the number of EEG samples in normal cases. FP is false positive results represent incorrect classifications of the negative cases into positive class. FN is false negative results denote incorrect classifications of positive cases into negative class. They ACC, SEN, SPE have been calculated as follow:

$A C C=(T P+T N) / N$

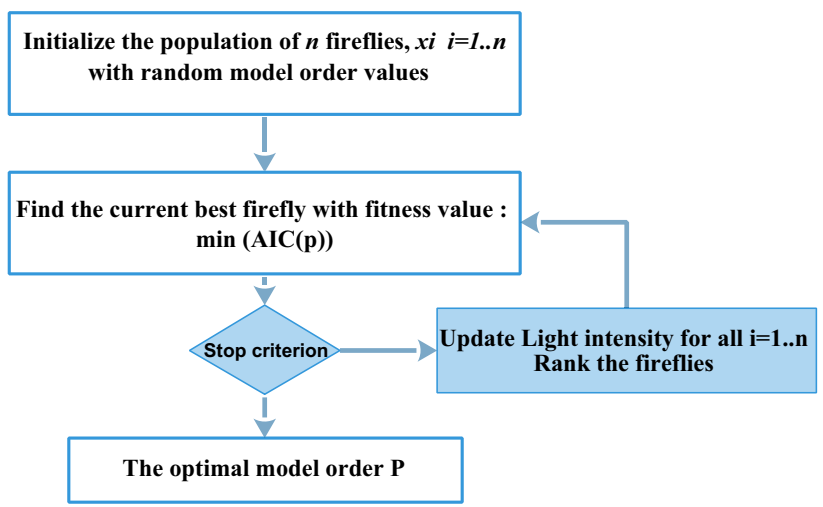

Fig. 2 Flowchart for AR model order P with FA algorithm 

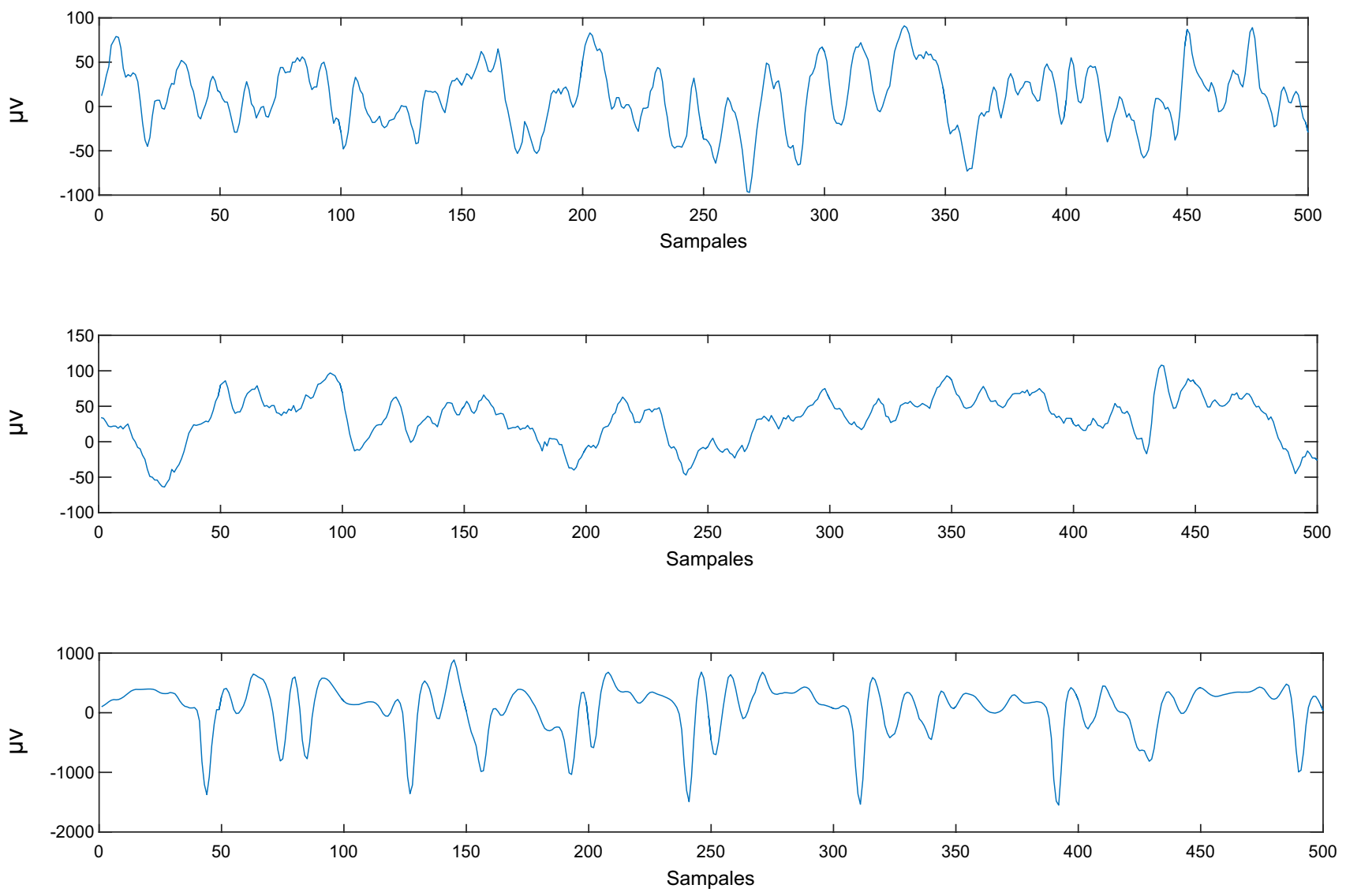

Fig. 3 Exemplary typical EEG signals of the sets. Class (Z, O), class (N, F), and class(S)

$S E N=T P /(T P+F N)$

$\mathrm{SPE}=\mathrm{TN} /(\mathrm{TN}+\mathrm{FP})$

Every signal has been divided onset of segments $23.6 \mathrm{~s}$. The notation A,B,C,D,E have been employed for the classes $\mathrm{Z}, \mathrm{O}, \mathrm{N}, \mathrm{F}, \mathrm{S}$, respectively, in order to make comparison with other works. In the experiments, the best model order P has been selected by using the AIC metric for Burg AR model. The final classification has been studied as follows: (i) healthy no-epileptic (Normal) to Interictal (sets A, B) to (sets C, D), (ii) healthy no-epileptic (Normal) to Ictal (sets A, B) to (set E), (iii) Interictal To Ictal (sets C, D) to (set E) (see Fig. 4).

\subsection{Experimental results}

\subsubsection{EEG the features extraction using AR model without FA method}

Table 1 summarized this classification. The average of performance measure is given in Table 2. It can be seen from Table 1 that the highest classification accuracies of Sets (A and E)and (B and E) are $98.00 \%$ for both. Moreover, the

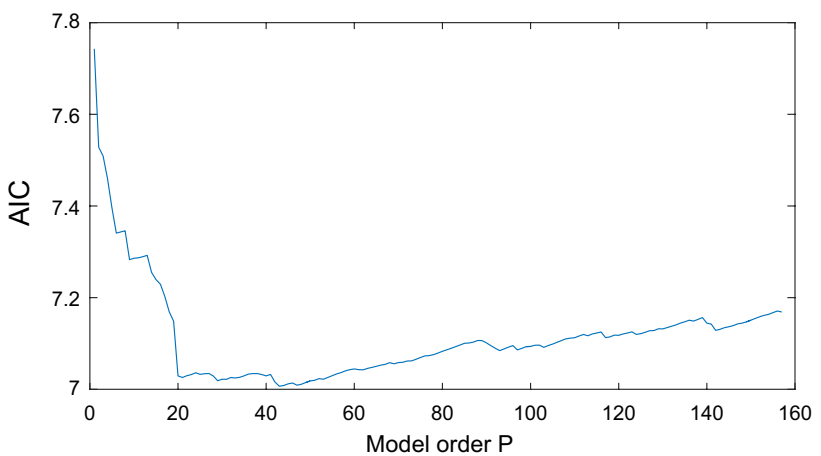

Fig. 4 Illustrates example of the $\mathrm{AIC}(\mathrm{P})$ variation

classification accuracies for the rest of the pairs of Sets are between $94.00 \%$ and $96.00 \%$. It is also easy to see that the proposed method can achieve an average classification of $98.0 \%, 100.00 \%$ and $96.00 \%$ of accuracy, sensitivity and specificity, respectively, for healthy no-epileptic (Normal) to Interictal. 
Table 1 Results of classification using (Burg and SVM))

\begin{tabular}{|c|c|c|c|c|c|c|c|c|}
\hline & \multicolumn{3}{|c|}{ Healthy no-epileptic (Normal) set A } & \multicolumn{3}{|c|}{ Healthy no-epileptic (Normal) set B } & \multirow{2}{*}{\multicolumn{2}{|c|}{$\begin{array}{l}\text { Interictal (sets C, } \\
\text { D) to ictal (set E) }\end{array}$}} \\
\hline & \multicolumn{2}{|c|}{$\begin{array}{l}\text { To interictal (sets } \\
\text { C, D) }\end{array}$} & \multirow{2}{*}{$\begin{array}{l}\text { To Ictal set } \mathrm{E} \\
\text { AE }\end{array}$} & \multicolumn{2}{|c|}{$\begin{array}{l}\text { To Interictal } \\
\text { (sets C,D) }\end{array}$} & \multirow{2}{*}{$\begin{array}{l}\text { To Ictal set E } \\
\text { BE }\end{array}$} & & \\
\hline & $\mathrm{AC}$ & $\mathrm{AD}$ & & $\mathrm{BC}$ & $\mathrm{BD}$ & & $\mathrm{CE}$ & $\mathrm{DE}$ \\
\hline ACC & 0.9600 & 0.9500 & 0.9800 & 0.9400 & 0.9600 & 0.9800 & 0.9500 & 0.9500 \\
\hline SEN & 0.9400 & 0.9800 & 1.0000 & 0.9000 & 0.9600 & 1.0000 & 0.9400 & 0.9400 \\
\hline SPE & 0.9800 & 0.9200 & 0.9600 & 0.9800 & 0.9600 & 0.9600 & 0.9600 & 0.9600 \\
\hline
\end{tabular}

\begin{tabular}{llcl}
\hline & $\begin{array}{l}\text { Healthy no-epileptic (Normal) to } \\
\text { interictal (\%) }\end{array}$ & $\begin{array}{l}\text { Healthy no-epileptic (Normal) to } \\
\text { ictal }(\%)\end{array}$ & $\begin{array}{l}\text { Interictal (sets C, D) } \\
\text { to ictal (set E) (\%) }\end{array}$ \\
\hline ACC & 95.25 & 98.00 & 95.00 \\
SEN & 94.50 & 100.00 & 94.00 \\
SPE & 96.00 & 96.00 & 96.00 \\
\hline
\end{tabular}

Table 2 The average of performance measure of the first experiment (AR burg SVM) and E). Also, in the second experiment (Burg with AR-FA and SVM), the proposed framework attained an excellent performance of $100.00 \% 100.00 \% 100.00 \%$ for accuracy, sensitivity, specificity, respectively, in the sets (A and E). In Table 5 we can notice that the best reported result is $100 \%$ of this work on the data set (A and E) and also $97.00 \%$ on (A and D).All in all, the presented technique outperformed previously proposed methods for epileptic seizures data classification.

\section{Conclusion}

In this paper, an automated framework has been proposed to classify and detect epileptic seizures from EEG signals by combining autoregressive model (AR) and Firefly Optimization to produce an optimal model order $(\mathrm{P})$ with support vector machines for high performances. Two experimental directions were followed on public Bonn dataset. In first direction, the features were extracted using AR burg model then SVM classifier. While in the second direction, the model order $\mathrm{P}$ is selected by FA method to be input to the AR burg model to generate the best coefficients then SVM classifier. Proposed method is able to achieve a good average of classifications accuracies between 94.50 and $100 \%$. Moreover, proposed framework outperformed previous studies reported in literatures. The focus of the future work is to integrate other AR model with burg method such as ARIMA algorithm to fuse these AR models in the features extraction in order to enhance the accuracy of classification and diagnosis of epilepsy. 
Fig. 5 Some examples of the changes of AIC fitness value with FA algorithm: a for the changes of AIC fitness value set $\mathrm{A} ; \mathbf{b}$ for the changes of AIC fitness value set $\mathrm{C}$; $\mathbf{c}$ for the changes of AIC fitness value set $\mathrm{E}$

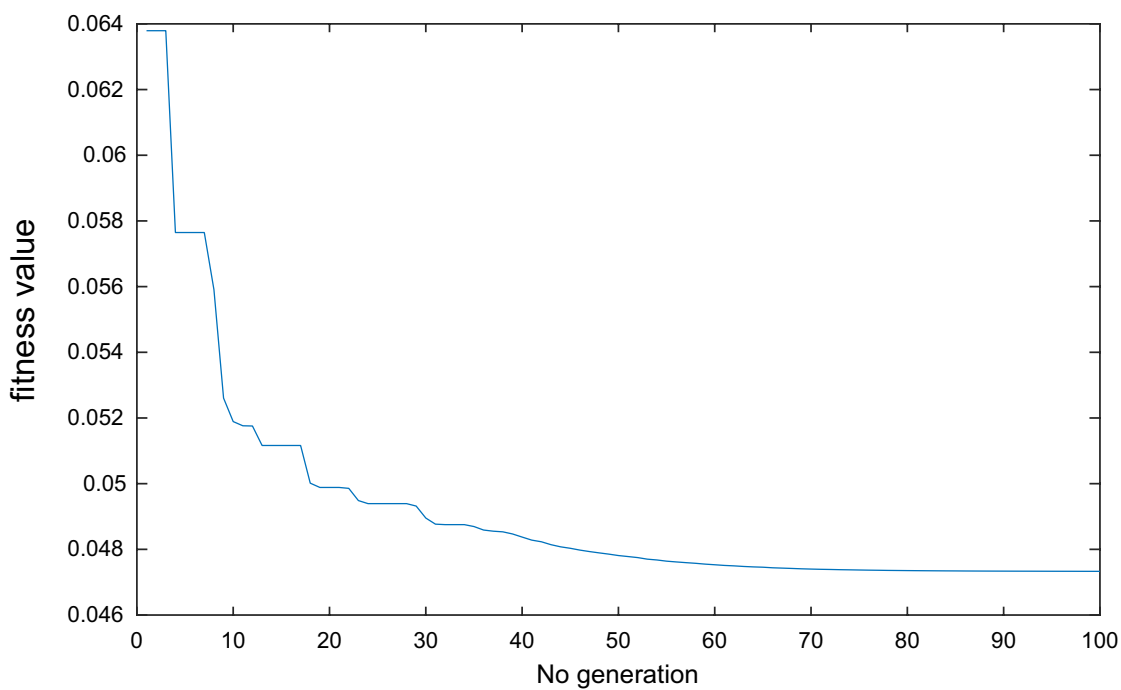

(a)

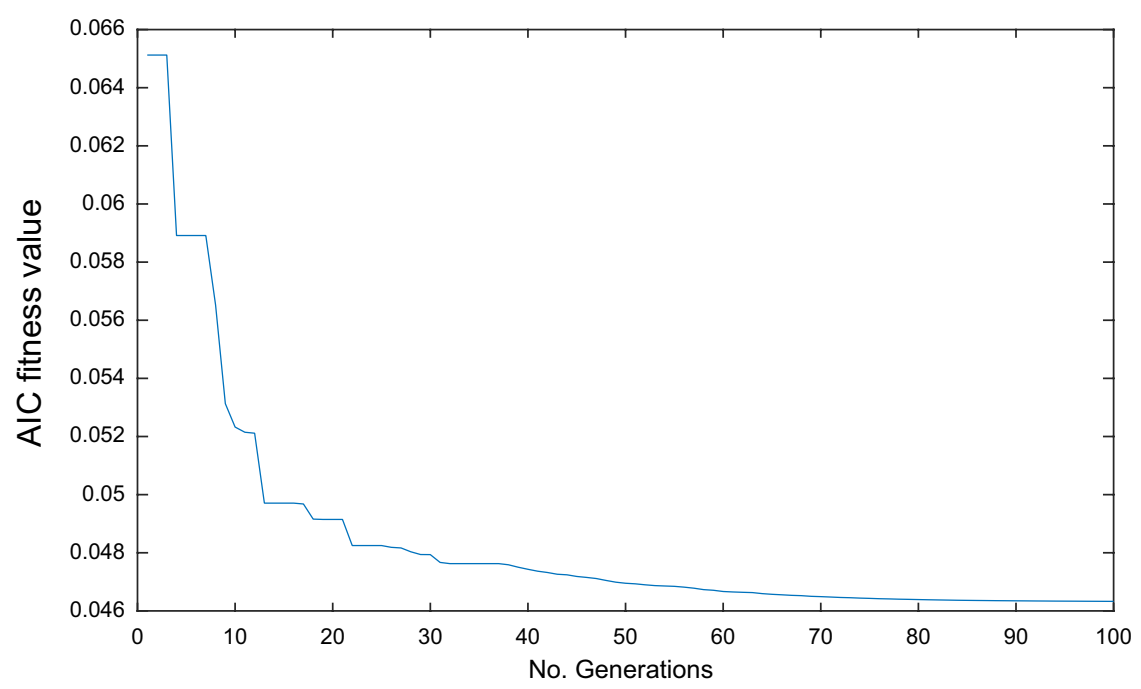

(b)

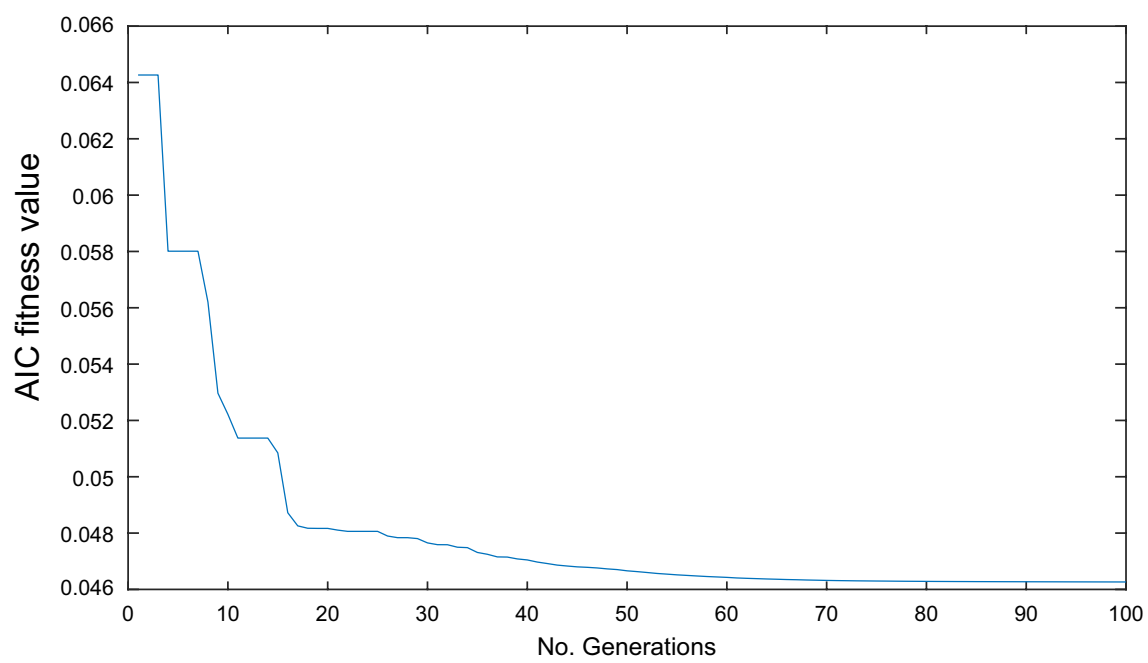

(c) 
Table 3 The obtained classification results by the second experiment (AR-FA SVM)

\begin{tabular}{|c|c|c|c|c|c|c|c|c|}
\hline & \multicolumn{3}{|c|}{$\begin{array}{l}\text { Healthy no-epileptic (Normal) (set } \\
\text { A) to interictal (sets C, D) and to } \\
\text { Ictal (set E) }\end{array}$} & \multicolumn{3}{|c|}{$\begin{array}{l}\text { Healthy no-epileptic (Normal) (set } \\
\text { B) to Interictal (sets C, D) and to } \\
\text { Ictal (set E) }\end{array}$} & \multicolumn{2}{|c|}{$\begin{array}{l}\text { Interictal (sets C, } \\
\text { D) To Ictal (set E) }\end{array}$} \\
\hline & $\mathrm{AC}$ & $\mathrm{AD}$ & $\mathrm{AE}$ & $\mathrm{BC}$ & BD & $\mathrm{BE}$ & $\mathrm{CE}$ & $\mathrm{DE}$ \\
\hline $\mathrm{ACC}$ & 0.9600 & 0.9700 & 1.0000 & 0.9400 & 0.9700 & 0.9900 & 0.9900 & 0.9500 \\
\hline SEN & 1.0000 & 0.9400 & 1.0000 & 0.9200 & 0.9400 & 1.0000 & 1.0000 & 0.9400 \\
\hline SPE & 0.9200 & 1.0000 & 1.0000 & 0.9600 & 1.0000 & 1.0000 & 0.9800 & 0.9600 \\
\hline
\end{tabular}

\begin{tabular}{llcl}
\hline & $\begin{array}{l}\text { Healthy no-epileptic (Normal) to } \\
\text { Interictal (\%) }\end{array}$ & $\begin{array}{l}\text { Healthy no-epileptic (Normal) } \\
\text { to Ictal (\%) }\end{array}$ & $\begin{array}{l}\text { Interictal to } \\
\text { Ictal }(\%)\end{array}$ \\
\hline ACC & 96,00 & 99.50 & 97.00 \\
SEN & 96.50 & 100.00 & 97.00 \\
SPE & 96.50 & 100.00 & 97.00 \\
\hline
\end{tabular}

Table 4 The average of performance measure of the second experiment (AR-FA SVM)

Table 5 Accuracies comparison between the introduced methods with other methods given in the literature

\begin{tabular}{|c|c|c|c|}
\hline Authors & Dataset & Methods & Accuracy $(\%)$ \\
\hline \multirow[t]{6}{*}{ This work } & \multirow[t]{2}{*}{$\mathrm{A}$ and $\mathrm{E}$} & Burg + SVM & 98.00 \\
\hline & & $\mathrm{AR}-\mathrm{FA}+\mathrm{SVM}$ & 100 \\
\hline & \multirow[t]{2}{*}{ A and D } & Burg + SVM & 95.00 \\
\hline & & $\mathrm{AR}-\mathrm{FA}+\mathrm{SVM}$ & 97.00 \\
\hline & \multirow[t]{2}{*}{$(\mathrm{C}$ and $\mathrm{D})$ and $(\mathrm{E})$} & Burg + SVM & 95.00 \\
\hline & & $\mathrm{AR}-\mathrm{FA}+\mathrm{SVM}$ & 97.00 \\
\hline Swami et al. (2016) & $\mathrm{A}$ and $\mathrm{E}$ & GRNN & 100 \\
\hline Guo et al. (2010) & $\mathrm{A}$ and $\mathrm{E}$ & ANN & 99.66 \\
\hline \multirow[t]{3}{*}{ Subasi and Gursoy (2010) } & \multirow[t]{3}{*}{$\mathrm{A}$ and $\mathrm{E}$} & $\mathrm{SVM}+\mathrm{PCA}$ & 98.75 \\
\hline & & SVM + ICA & 99.50 \\
\hline & & SVM + LDA & 100 \\
\hline Chandaka et al. (2009) & $\mathrm{A}$ and $\mathrm{E}$ & $\begin{array}{l}\text { Cross-correlation aided SVM classifier } \\
\text { signal }\end{array}$ & 95.96 \\
\hline \multirow[t]{2}{*}{ Nicolaou and Georgiou (2012) } & A and $\mathrm{E}$ & \multirow[t]{2}{*}{ Permutation Entropy (PE) and SVM } & 93.55 \\
\hline & A and D & & 88.83 \\
\hline \multirow[t]{2}{*}{ Yalcin et al. (2015) } & \multirow[t]{2}{*}{$\mathrm{A}$ and $\mathrm{E}$} & PSO-based neural network PSONN3 & 99.67 \\
\hline & & PSO-based neural network PSONN4 & 98.75 \\
\hline
\end{tabular}

\section{References}

Akaike H (1974) A new look at the statistical model identification. IEEE Trans Autom Control 19(6):716-723

Amorim P, Moraes T, Fazanaro D, Silva J, Pedrini H (2017) Electroencephalogram signal classification based on shearlet and contourlet transforms. Expert Syst Appl 67:140-147

Andrzejak RG, Lehnertz K, Mormann F, Rieke C, David P, Elger CE (2001) Indications of nonlinear deterministic and finite-dimensional structures in time series of brain electrical activity: dependence on recording region and brain state. Phys Rev E 64(6):61907

Angelov P, Kasabov N (2006) Evolving intelligent systems, eIS. IEEE SMC eNewsLett 15:1-13

Angelov P, Zhou X (2008) On line learning fuzzy rule-based system structure from data streams. In: IEEE International Conference on fuzzy systems (IEEE World Congress on Computational Intelligence), 2008, pp 915-922

Ansari M, Othman F, Abunama T, El-Shafie A (2018) Analysing the accuracy of machine learning techniques to develop an integrated influent time series model: case study of a sewage treatment plant, Malaysia. Environ Sci Pollut Res 25(12):12139-12149

Attia A, Moussaoui A, Chahir Y (2017) An EEG-fMRI fusion analysis based on symmetric techniques using dempster shafer theory. J Med Imaging Health Inf 7(7):1493-1501

Atyabi A, Luerssen MH, Fitzgibbon SP, Powers DMW (2012) The impact of PSO based dimension reduction on EEG classification. In: International Conference on brain informatics, 2012, pp 220-231

Belhadj S, Attia A, Adnane BA, Ahmed-Foitih Z, Ahmed AT (2016a) A novel epileptic seizure detection using fast potential-based hierarchical agglomerative clustering based on emd. IJCSNS 16(5):7 
Belhadj S, Attia A, Adnane AB, Ahmed-Foitih Z, Taleb AA (2016) Whole brain epileptic seizure detection using unsupervised classification. In: 2016 8th International Conference on modelling, identification and control (ICMIC), 2016, pp 977-982

Burg JP (1968) A new analysis technique for time series data. In: Pap. Present. NATO Adv. Study Inst. Signal Process. Enschede, Netherlands

Byun H, Lee S-W (2002) Applications of support vector machines for pattern recognition: A survey. In: Pattern recognition with support vector machines. Springer, pp 213-236

Chandaka S, Chatterjee A, Munshi S (2009) Cross-correlation aided support vector machine classifier for classification of EEG signals. Expert Syst Appl 36(2):1329-1336

Chiarelli AMM, Zappasodi F, Di Pompeo F, Merla A (2017) Simultaneous functional near-infrared spectroscopy and electroencephalography for monitoring of human brain activity and oxygenation: a review. Neurophotonics 4(4):41411

Cortes C, Vapnik V (1995) Support-vector networks. Mach Learn 20(3):273-297

Erguzel TT, Ozekes S, Tan O, Gultekin S (2015) Feature selection and classification of electroencephalographic signals: an artificial neural network and genetic algorithm based approach. Clin EEG Neurosci 46(4):321-326

Fabri SG, Camilleri KP, Cassar T (2011) .Parametric modelling of EEG data for the identification of mental tasks. In: Biomedical engineering, trends in electronics, communications and software. InTech

Guo L, Rivero D, Dorado J, Rabunal JR, Pazos A (2010) Automatic epileptic seizure detection in EEGs based on line length feature and artificial neural networks. J Neurosci Methods 191(1):101-109

Krusienski DJ, McFarland DJ, Wolpaw JR (2006) An evaluation of autoregressive spectral estimation model order for brain-computer interface applications. In: Engineering in Medicine and Biology Society, 2006. EMBS'06. 28th Annual International Conference of the IEEE, 2006, pp 1323-1326

Khushaba RN, Al-Ani A, Al-Jumaily A, Nguyen HT (2008) A hybrid nonlinear-discriminant analysis feature projection technique. In: Australasian Joint Conference on artificial intelligence, 2008, pp $544-550$

Nicolaou N, Georgiou J (2012) Detection of epileptic electroencephalogram based on permutation entropy and support vector machines. Expert Syst Appl 39(1):202-209

Ordóñez C, Lasheras FS, Roca-Pardiñas J, de Cos Juez FJ (2019) A hybrid ARIMA-SVM model for the study of the remaining useful life of aircraft engines. J Comput Appl Math 346:184-191

Ouelli A, Elhadadi B, Aissaoui H, Bouikhalene B (2015) Epilepsy seizure detection using autoregressive modelling and multiple layer perceptron neural network. Am J Comput Sci Eng 2(4):26-31
Padmavathi K, Ramakrishna KS (2015a) Classification of ECG signal during atrial fibrillation using autoregressive modeling. Proc Comput Sci 46:53-59

Padmavathi K, Ramakrishna KS (2015b) Classification of ECG signal during atrial fibrillation using Burg's method. Int J Electr Comput Eng 5(1):64

Sharma R, Pachori RB (2015) Classification of epileptic seizures in EEG signals based on phase space representation of intrinsic mode functions. Expert Syst Appl 42(3):1106-1117

Shiman F, Safavi SH, Vaneghi FM, Oladazimi M, Safari MJ, Ibrahim F (2012) EEG feature extraction using parametric and non-parametric models. In: Biomedical and Health Informatics (BHI). IEEE-EMBS International Conference on 2012:66-70

Subasi A, Gursoy MI (2010) EEG signal classification using PCA, ICA, LDA and support vector machines. Expert Syst Appl 37(12):8659-8666

Swami P, Gandhi TK, Panigrahi BK, Tripathi M, Anand S (2016) A novel robust diagnostic model to detect seizures in electroencephalography. Expert Syst Appl 56:116-130

Tezel G et al (2009) A new approach for epileptic seizure detection using adaptive neural network. Expert Syst Appl 36:172-180

Übeyli ED (2008a) Analysis of EEG signals by combining eigenvector methods and multiclass support vector machines. Comput Biol Med 38(1):14-22

Übeyli ED (2008b) Analysis of EEG signals by combining eigenvector methods and multiclass support vector machines. Comput Bio. Med 38(1):14-22

Übeyli ED (2010) Least squares support vector machine employing model-based methods coefficients for analysis of EEG signals. Expert Syst Appl 37(1):233-239

Wang D, Miao D, Xie C (2011) Best basis-based wavelet packet entropy feature extraction and hierarchical EEG classification for epileptic detection. Expert Syst Appl 38(11):14314-14320

Yalcin N, Tezel G, Karakuzu C (2015) Epilepsy diagnosis using artificial neural network learned by PSO. Turk J Electr Eng Comput Sci 23(2):421-432

Yang X-S (2009) Firefly algorithms for multimodal optimization. In: International symposium on stochastic algorithms, pp 169-178

Zhang Y, Liu B, Ji X, Huang D (2017) Classification of EEG signals based on autoregressive model and wavelet packet decomposition. Neural Process Lett 45(2):365-378 